\title{
Correction to: Shared Individual Formulation Therapy (SIFT): an open-label trial of a new therapy accommodating patient heterogeneity in functional neurological disorder
}

\author{
M. Gutkin ${ }^{1,2}$ - R. J. Brown ${ }^{3,4} \cdot$ L. McLean ${ }^{2,5} \cdot$ J. Streimer ${ }^{2} \cdot$ R. A. Kanaan ${ }^{1}$
}

Published online: 5 October 2021

๑) Springer-Verlag GmbH Germany, part of Springer Nature 2021

\section{Correction to: Journal of Neurology https://doi.org/10.1007/s00415-021-10797-y}

The original version of this article unfortunately contained a mistake in Fig. 4.

The corrected Fig. 4 given below:

Fig. 4 Frequency of exit interview rating scales

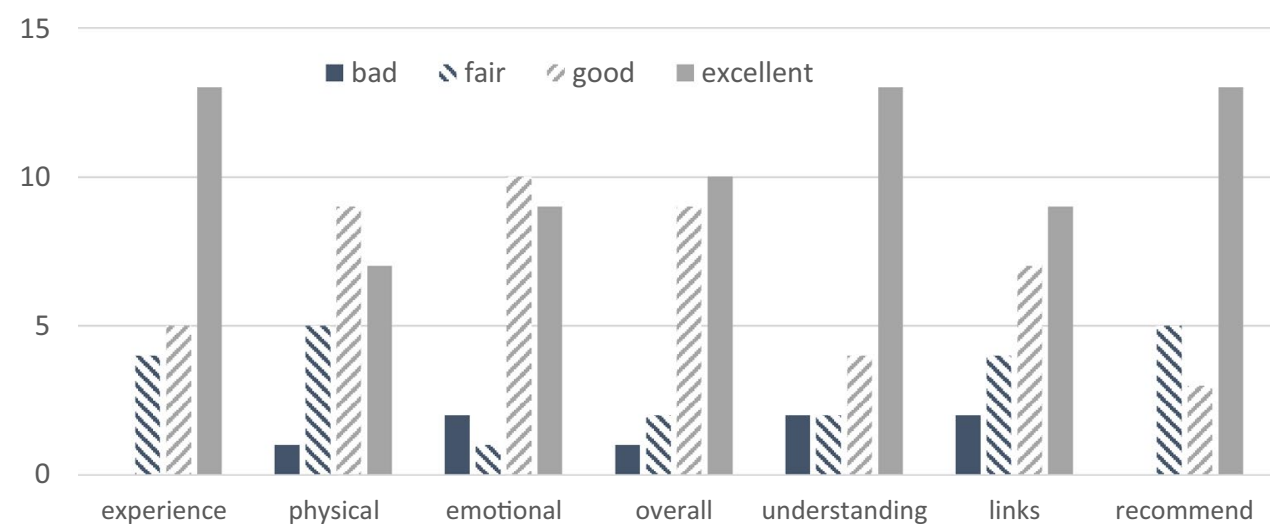

The original article can be found online at https://doi.org/10.1007/ s00415-021-10797-y.

\section{Gutkin}

myles.gutkin@unimelb.edu.au

1 Department of Psychiatry, The University of Melbourne, Austin Health, Heidelberg, Victoria, Australia

2 Department of Consultation-Liaison Psychiatry, Royal North Shore Hospital, St Leonards, New South Wales, Australia

3 Division of Psychology and Mental Health, The University of Manchester, Manchester, UK

4 Greater Manchester Mental Health NHS Foundation Trust, Manchester, UK

5 Brain and Mind Centre, The University of Sydney, Camperdown, New South Wales, Australia 